\title{
Sub-surface molecular analysis and imaging in turbid media by time-gated Raman spectral multiplexing
}

\author{
C. Corden ${ }^{1}$, P Matousek ${ }^{2}, \mathrm{C} \mathrm{Conti}^{3}$, I Notingher ${ }^{1, *}$ \\ ${ }^{1}$ School of Physics and Astronomy, University of Nottingham, University Park, Nottingham \\ NG7 2RD, United Kingdom. \\ ${ }^{2}$ STFC Rutherford Appleton Laboratory, Central Laser Facility, Research Complex at Harwell, \\ Harwell Oxford OX11 0QX, United Kingdom. \\ ${ }^{3}$ Institute of Heritage Science, National Research Council, Via Cozzi 53, Milano, Italy. \\ * Corresponding author: Ioan.notingher@nottingham.ac.uk.
}

\begin{abstract}
Obtaining molecular information deeper within optically-turbid samples is valuable in many applications. However, in many cases this is challenging, in particular when the sample elicits strong laser-induced fluorescence emission. Here, we investigated the use of time-gated and micro-spatially-offset Raman spectroscopy (micro-SORS) based on spectral multiplexing detection to obtain subsurface molecular analysis and imaging for both fluorescing and nonfluorescing samples. The multiplexed spectral detection achieved with a digital micromirror device (DMD) allowed fast acquisition of the time-gated signals to enable 3D Raman mapping (raster scanning in the lateral $x-y$ plane and using time-of-flight calibration for the axial $z$ direction). Sub-millimetre resolution molecular depth mapping was achieved with dwell times on the order of seconds per pixel. To suppress fluorescence backgrounds and enhance Raman bands, time-gated Raman spectroscopy was combined with micro-SORS to recover Raman signals of red pigments placed behind a layer of optically turbid material. Using a defocusing micro-SORS approach, both fluorescence and Raman signals from the surface layers were further suppressed, which enhanced the Raman signals from the deeper sublayers containing the pigment. These results demonstrate that time-gated Raman spectroscopy based on spectral multiplexed detection, and in combination with micro-SORS, is a powerful technique for subsurface molecular analysis and imaging, which may find practical applications in medical imaging, Cultural Heritage, forensics, and industry.
\end{abstract}

\section{Introduction}

Raman spectroscopy is a powerful non-destructive technique for molecular analysis. ${ }^{1}$ For many samples, the regions of interest lie beneath a surface layer. If the sample is transparent, confocal Raman microscopy can measure depth-related molecular information with high accuracy. ${ }^{2-4}$ However, for samples eliciting a high level of light scattering, probing deeper in the sample is challenging as the Raman photons are diffused and attenuated. Furthermore, many samples of interest, such as those often encountered in Cultural Heritage or medical applications are difficult to analyse due to intense laser-induced fluorescence emission, which can overlap and potentially swamp the Raman bands.

Time-gated Raman spectroscopy has been shown to be effective for both depth analysis of optically turbid samples ${ }^{5-7}$ and suppression of fluorescence backgrounds. ${ }^{8-12}$ Molecular depth 
information can be determined by measuring the time of flight of scattered Raman photons. Through an optically transparent sample, light travels at a speed $c / n$, where $\mathrm{c}$ is the speed of light in vacuum and $\mathrm{n}$ is the refractive index of the samples (e.g. $\sim 4.5 \mathrm{ps}$ for $1 \mathrm{~mm}$ of water), which is shorter than the temporal resolution of current time-gated Raman spectroscopy instruments. However, light propagating through an optically turbid sample is diffusely scattered and has been shown to take 10-20 times longer to propagate ${ }^{13}$, allowing for submillimetre resolution depth analysis using current technologies.

Due to various technical limitations, the detection efficiency of current time-gated Raman spectroscopy instruments is significantly less than for an optimized steady-state CCD based spectrometer. Furthermore, many time-gated Raman instruments operate with a fixed gate position. Therefore, to analyse the Raman intensity as a function of time, multiple measurements must be made while shifting the gate window with respect to the excitation laser pulse. As a consequence, subsurface 3-D Raman imaging by time-gated Raman spectroscopy has been impractical. By operating in time correlated single photon counting mode (TCSPC), all photons are collected, time tagged and stored to memory in a single measurement window, significantly improving acquisition time.

Digital light processing technologies such as digital micromirror devices (DMD) have recently reached an efficiency level that meets the most stringent requirements of Raman instruments. Their integration allows for real time manipulation of optical systems, paving the way for a range of techniques that could not be alternatively achieved ${ }^{14}$. For example, dispersing a spectrum across a DMD allows multiple wavelengths to be simultaneously focused onto a single detector (i.e. spectral multiplexing), essentially compressing the data at the measurement stage, hence these techniques have become known as compressive sensing ${ }^{15-18}$. DMDs have recently been implemented to generate software-configurable, reflective pinhole arrays for Raman spectroscopy applications, including multi-foci confocal Raman spectroscopy with combined optical tweezers ${ }^{3,19-22}$ and spatially offset Raman spectroscopy (SORS) with software configurable collection points. ${ }^{23-25}$

For an optically turbid material, subsurface photons undergo multiple scattering events. The diffused photons follow a random path and exit the material at a range of positions with respect to the excitation point. In a backscattering geometry, SORS exploits this effect in order to collect diffused Raman photons from subsurface regions. This is achieved by spatially introducing an offset between the laser illumination and Raman collection points. ${ }^{26}$ Such offsets can be implemented by using optical fibres ${ }^{27}$, DMDs ${ }^{24}$, or using a CCD ${ }^{28}$. MicroSORS (combining microscopy with SORS) has recently been shown to be effective in enhancing the Raman spectrum of the deeper, non-fluorescing target sublayer to be acquired. ${ }^{30}$ However, micro-SORS cannot measure the Raman bands of a sample that elicits strong laser induced florescence because SORS cannot discriminate between fluorescence and Raman photons.

In this paper we use raster scanning time-gated Raman spectroscopy based on spectral multiplexed detection to obtain 3-D Raman information from optically turbid samples. In this approach, the spectral multiplexing works as a compressive sensing technique, that has been used in continuous wave Raman spectroscopy to speed up imaging. ${ }^{15,31-33}$ This technique has also been applied recently to time-gated Raman spectroscopy in order to decrease the acquisition times and enable time-gated Raman mapping of samples eliciting strong fluorescence backgrounds. ${ }^{34}$ Using a single pixel detector, such as a single photon avalanche diode (SPAD), operating in TCSPC mode combined with multiplexing the Raman bands of interest, provides a speed advantage and higher signal to noise ratio. For optically turbid samples eliciting strong laser-induced fluorescence emission, time-gated Raman spectroscopy was combined with micro-SORS to achieve sub-surface Raman analysis with sufficient suppression of the fluorescence background. Micro-SORS allows suppression of the signal 
from the surface layer, while the time-gated detection provides the suppression of the fluorescence background of the deeper target layer. While time gating alone can suppress signal from the surface layer, a certain degree of temporal overlap between the different layers still remains, resulting in the detection of unwanted surface generated photons at longer time gates. While for non-fluorescing samples this can be often solved by subtraction or other data methods, for fluorescing samples such overlap is undesirable, leading to strong background signal (shot noise), thus further suppression is required. We present experimental results using layered, non-fluorescing polymer samples and use a range of fluorescing pigments/dyes and substrate materials to emphasise the potential use of the time gated micro-SORS technique for Cultural Heritage applications. In fact, fluorescence is one of the main obstacles often encountered in Cultural Heritage materials, as they exhibit a complex mixture of organic and inorganic compounds, frequently damaged and transformed due to the aging and decay mechanisms.

\section{Materials and Methods}

A schematic description of the instrument is presented in the Supplemental material (Figure S1). A $775 \mathrm{~nm}$ wavelength, $30 \mathrm{ps}, 5 \mathrm{~nJ}$ pulsed laser with $10 \mathrm{MHz}$ repetition rate (Katana, NKT photonics, Denmark) is used to excite the sample using a Nikon Eclipse inverted microscope via a 0.75 NA, 20x Nikon objective. The backscattered Raman photons are collected, and fibre coupled into an Andor Shamrock 303i spectrometer, where the spectrum can be either be focused onto an Andor DU401 CCD for general continuous wave (CW) Raman spectroscopy, or focused upon a Texas Instruments digital micromirror device (DMD, Texas instruments DLPLCR4500EVM)) for wavelength selection. The reflected spectrum from the DMD is then focused through the entry port of a second spectrometer (Shamrock 303i) to recombine the spectral features into a collimated polychromatic beam. This collimated beam can then be efficiently focused onto a $100 \mu \mathrm{m}$ SPAD detector (MPD 100CTE (dark count rate < 30 counts per second)). The DMD is programmed to direct the light corresponding to the spectral features of interest to the SPAD, whose output is coupled to a high performance time to digital convertor where it is then compared with the synchronization pulse from the laser. All photons detected are assigned a time tag before being streamed to a $\mathrm{PC}$ via a microcontroller. An instrument response function of $60 \mathrm{ps}$ full width half $\max (\mathrm{FWHM})$ was determined by measuring the Raman signal from an optically clear, non-fluorescing sample. Custom software using MATLAB was used for serial communication, data acquisition and analysis. A slave microcontroller (Atmel SAM3X8E ARM Cortex-M3) is programmed to handle the SPI communication to the time to digital convertor and to send and receive data to MATLAB.

The instrument can be used in two main modes of operation, as described previously: ${ }^{34}$ In the "DMD scanning mode", the Raman spectrum is reconstructed by sequentially scanning the micro-mirrors of the DMD. This mode is slow but allows the measurement of Raman spectra of unknown samples. Here the DMD was programmed to scan a rectangular array of mirrors analogous to a slit width of $100 \mu \mathrm{m}$. In total 150 steps were used to build up the full spectrum, with a $20 \mathrm{~s}$ integration time per step (total $3000 \mathrm{~s}$ per spectrum).

The "Spectral multiplexing mode" is used when the spectral features of the sample are known. In this case, the DMD can be programmed to use only selected micro-mirrors to direct multiple spectral bands to the SPAD (multiplexed detection). For the multiplexed depth analysis, the Raman maps were generated with a total dwell time of $16 \mathrm{~s}$ per pixel $(7.1 \mathrm{hrs}$ for a $40 \mathrm{x} 40$ pixel map).

Micro-SORS measurements were carried out using the defocusing variant, consisting in the acquisition of a series of Raman spectra at imaged (focused) position and displacing away the 
sample surface from the microscope objective as described previously. ${ }^{35}$ The enlargement of the laser illumination and Raman collection areas allows achieving a higher relative content of the sublayer._Here, two defocused positions were applied, $-200 \mu \mathrm{m}$ and $-400 \mu \mathrm{m}$, using a Nikon 20x 0.75 NA microscope objective. The laser spot diameter is $\sim 8 \mu \mathrm{m}$ in focus, expanding to $\sim 210 \mu \mathrm{m}$ at a defocusing of $-400 \mu \mathrm{m}$. For 3D Raman mapping, the microscope objective was replaced by a lens with $40 \mathrm{~mm}$ focal length (equivalent to a $5 \mathrm{X}$ magnification) leading to an increase in the laser spot diameter to $\sim 30 \mu \mathrm{m}$. This configuration increases the depth of field for laser excitation and leads to an enhanced signal from the sublayer compared to the signal obtained with microscope objective.

Non-fluorescing samples: A set of calibration samples were prepared consisting of a $3 \mathrm{~mm}^{3}$ polystyrene (PS) cube sublayer behind Teflon surface layers of varying thicknesses. This was achieved by stacking multiple layers of $75 \mu \mathrm{m}$ thick polytetrafluoroethylene (PTFE/ Teflon) tape. Thicker layers of $1.5 \mathrm{~mm}$ and $3 \mathrm{~mm}$ layers of solid Teflon block (schematic in Figure 1a) were used as it was not practical to create such thick layers from multiple layers of thin sheets. Moreover, a sample consisting of two $3 \mathrm{~mm}^{3}$ polystyrene blocks placed behind Teflon layers of $0.6 \mathrm{~mm}$ and $1.5 \mathrm{~mm}$ thickness was prepared (see schematic in Figure $3 \mathrm{a}$ ) for 3-D Raman mapping.

Fluorescing samples: A selection of dyes and pigments used in art has been carried out on the basis of their fluorescence emission.

- Red lake dye (0207, Natural Red 25, C.I. 75450), purchased from "Zecchi Colori Belle Arti Restauro", and compressed into a $\mathrm{KBr}$ pellet (1 $\mathrm{mm}$ thickness, $14 \mathrm{~mm}$ diameter). A lac dye stated to be extracted from the insect coccus lacca as per suppliers's label, which infects fig trees indigenous to Asia and India. ${ }^{36}$ Used throughout history as far back as $250 \mathrm{AD}$ for dying of textiles, oil and water painting; Laccaic acid is the main chromophore.

- Carmine Naccarat pigment (42100, Natural Red 4, C.I. 75470), obtained from Kremer Pigmente, compressed into a $\mathrm{KBr}$ pellet $(1 \mathrm{~mm}$ thickness, $14 \mathrm{~mm}$ diameter $)$. An aluminium red lake of carminic acid, which is the principal chromophore. ${ }^{37}$ It is extracted from cochineal, a scale insect. Cochineal dye was used by the Aztecs and Mayas of Central and North America. It became one of Mexico's most valuable export goods during colonial times. ${ }^{38}$

- Quinacridone Red Magenta (23720, PV19, C.I. 73900), supplied by Kremer Pigmente, is the typical quinacridone dye (compressed into a $\mathrm{KBr}$ pellet (1 mm thickness, $14 \mathrm{~mm}$ diameter)). It is a relatively modern synthetic organic pigment first synthesized and used commercially in the 1950s and used extensively in the paint industry, particularly in automotive paints. Quinacridone pigments were used by artists soon after their commercial introduction and are sold to artists under a variety of names such as Rose Red, Violet, Cobalt Violet, Quinacridone Crimson, Quinacridone Gold, Quinacridone Red, Rose Violet and more. ${ }^{39}$

- Fluorescein dye (F2456, C.I. 45350:1), purchased from Sigma Aldrich is a highly fluorescent molecule widely used in chemistry, laser and solar energy fields and for wood, acrylic and canvas prints. It is also used for the preparation of colorants as eosin. Layered samples using fluorescein as a sub-surface layer were prepared: $1 \mathrm{~mm}^{3}$ of 
fluorescein powder, layered upon a $600 \mu \mathrm{m}$ thick layer of Teflon (eight layers of PTFE tape); $1 \mathrm{~mm}^{3}$ of fluorescein powder layered behind of a $100 \mu \mathrm{m}$ thick layer of highly fluorescent brown paper. Here, this sample was studied as a dry powder for convenience and proof of concept.

\section{Results}

\section{Time-gated Raman raster scanning for 3-D molecular imaging of non-fluorescing samples}

We investigated the ability of using time-gated Raman spectroscopy in spectral multiplexed detection mode to obtain 3-D Raman images of non-fluorescing optically turbid samples. This was achieved by raster scanning the sample in the lateral $(x-y)$ directions using the microscope translation stage and using the photon diffusion time to obtain the depth information in the axial direction ( $z$ axis).

First, samples consisting of two layers were used to understand the level of temporal overlap between the Raman photons originating from different depths of the sample (i.e. surface layer and deeper layer). For this, samples consisting of a $3 \mathrm{~mm}^{3}$ polystyrene (PS) cube covered by $1.5 \mathrm{~mm}$ and $3 \mathrm{~mm}$ thick sheets of Teflon were selected (schematic in Figure 1a). Time-gated Raman spectra of the sample consisting of the $1.5 \mathrm{~mm}$ thick Teflon surface layer were recorded in the DMD scanning mode and are presented in Figure 1b. The time-gated spectra were obtained by binning the data using 80 ps bin widths in 10 ps steps. Reference spectra of Teflon and polystyrene are also presented for reference, along with a range of different gate delays selected to illustrate the time evolution of the signal. For short time delays ( $40 \mathrm{ps})$ the sole contribution of the signal arises from the Teflon surface layer, as identified by the bands at $1216 \mathrm{~cm}^{-1}, 1299 \mathrm{~cm}^{-1}$ and $1379 \mathrm{~cm}^{-1}$. As the signal from the Teflon surface layer peaks (at around $60 \mathrm{ps}$ ) Raman bands assigned to polystyrene can be detected at low intensity, such as the $1001 \mathrm{~cm}^{-1}$ ring breathing vibration. As time progresses, a decrease in the signal from the Teflon surface layer and an increase in the polystyrene signal from the sublayer is observed. The experiments were repeated with a sample consisting of a $3 \mathrm{~mm}$ thick Teflon surface layer. To better understand the time evolution of the signals, the Raman bands for the Teflon and PS were integrated and the intensity plotted as a function of time (Figure 1c). The intensity versus time plots $I(t)$, in Figure 1c show a 100 ps time delay between the Teflon and polystyrene signal maxima when the $1.5 \mathrm{~mm}$ Teflon is used, which increases to $350 \mathrm{ps}$ when the thickness of Teflon layer is increased to $3 \mathrm{~mm}$.

A degree of temporal overlap is observed between the signals originating from different depths of the sample. For the $1.5 \mathrm{~mm}$ thick surface sample, the pure Raman spectrum from PS layer is obtained by subtraction of the Raman spectrum obtained at the shortest time-delay (e.g. 40 ps) from the Raman spectrum at a longer delay time (e.g. 1500 ps) (Figure 1d). The spectra were normalised and scaled accordingly to compensate for wavelength dependent scattering effects. 
a)

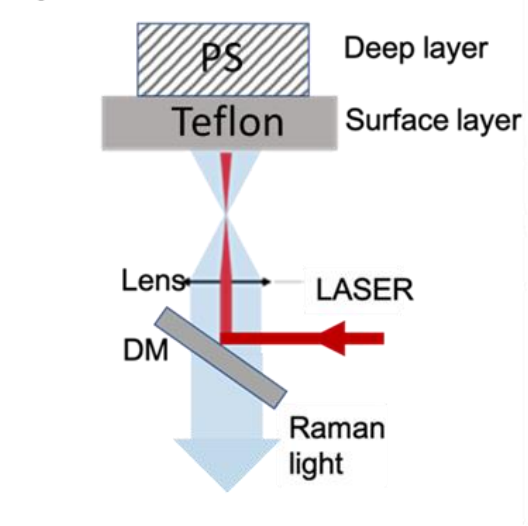

c)

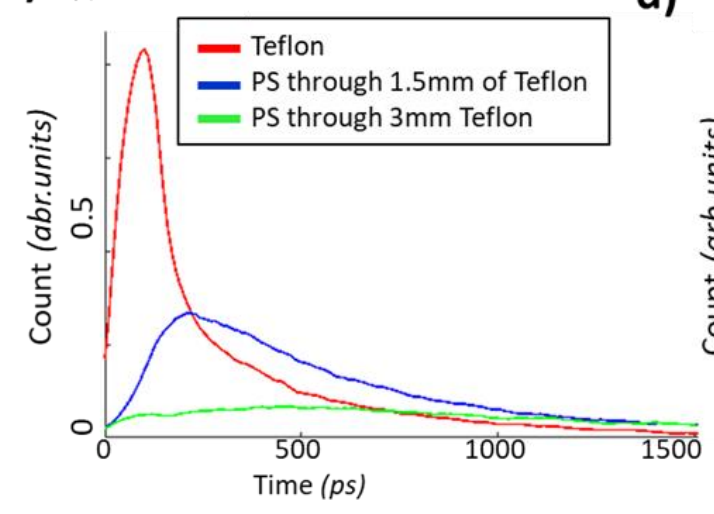

d) b)
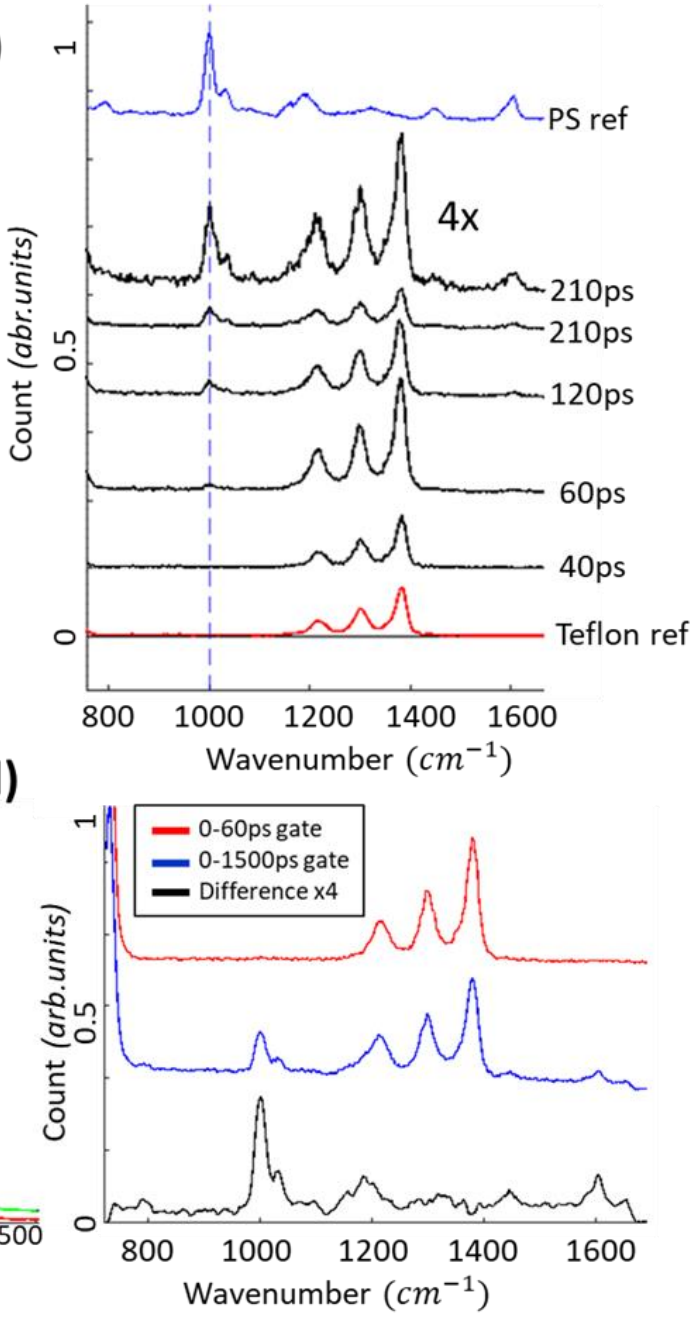

Figure 1. a) Schematic of a two-layered sample. b) Time gated Raman spectra polystyrene behind $1.5 \mathrm{~mm}$ of Teflon (red and blue reference spectra of Teflon and polystyrene). Spectra were offset vertically for clarity, but their intensity is not altered unless specified. c) Time histograms of signal from integration of the spectral features associated with polystyrene through 1.5 and $3 \mathrm{~mm}$ of Teflon sheet. d) $0-60$ ps gate time, $0-1500$ ps gate time and the difference (X4 for clarity).

After confirming the efficient detection of the Raman bands characteristic to Teflon and polystyrene (red and blue highlighted features in Figure 2a), the instrument was operated in spectral multiplexing mode in order to speed up the data acquisition. To provide a relationship between depth and diffusion time of the Raman photons, a set of calibration samples were prepared consisting of a polystyrene sublayer covered with Teflon surface layers of varying thicknesses. The Raman intensity of the polystyrene (through $n$ layers of Teflon) as a function of time is presented in Figure 2b. Analysis of the measured data provides a relationship between the thickness of the surface Teflon layer $(T(\mu \mathrm{m}))$ and photon diffusion time $(t(\mathrm{ps}))$, where the total diffusion time includes the excitation and radiated photons (the net photon migration distance is therefore twice the thickness). The time which corresponds to the maximum intensity of the polystyrene signal is taken as the net diffusion time (Figure 2b). This value was determined by taking the maximum value of a two- term exponential fit to the data (with 95\% confidence bounds) (an example fit is presented in the supplemental material Figure S4). The calculated values and associated errors are presented in Figure 2c. 
A linear fit to the data presented in Figure $2 \mathrm{c}$ provides the relationship between the diffusion time and the thickness of the Teflon: $T(\mu \mathrm{m})=8.53( \pm 1.5) * t(\mathrm{ps}) \pm 180 \mu \mathrm{m}$. Previous works suggest a constant photon migration rate specific to each material hence the decision to use a linear fit. ${ }^{13,40,41}$ However, the photon probability distribution will be weighted towards shorter distances as more scattering steps equates to a greater degree of attenuation; a more accurate model would therefore contain a decaying term to account for scattering losses. Furthermore, ideally, the calibration samples should be precisely machined from a single uniform block of material as there may be differences between the optical properties of bulk Teflon and stacked layers of PTFE tape. Although a more accurate calibration could be achieved, this level of accuracy was considered adequate here for the purpose of demonstrating proof-of-principle.
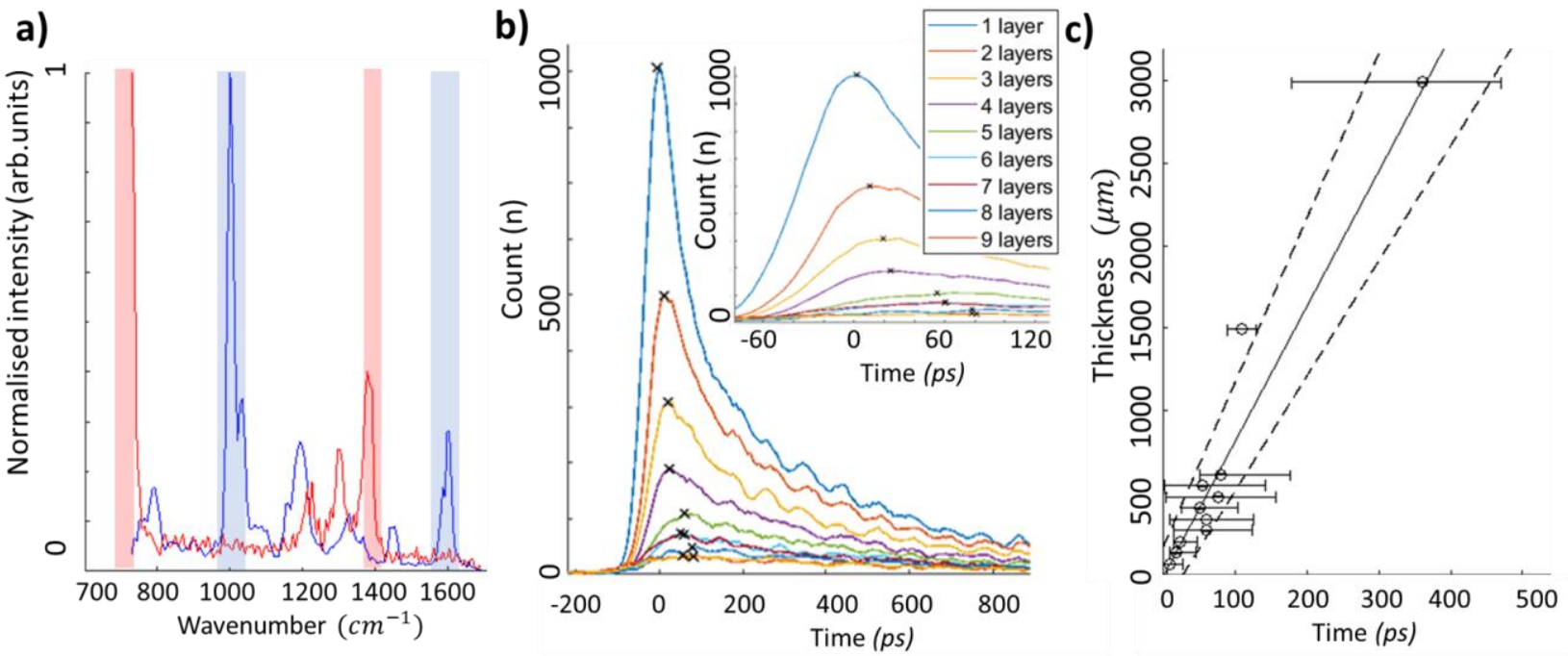

Figure 2. a) Reference spectra of Teflon and polystyrene with spectral features chosen for multiplexing highlighted in red and blue. b) Diffused Raman signals of polystyrene through n layers of PTFE tape plotted as a function of time $I(t)$, collected by multiplexing the polystyrene bands highlighted in Figure 2a (blue). c) Calibration of Teflon thickness vs diffusion time. Each data point and error bars were determined at the maximum of a two-term exponential fit to the data in Fig. 2b) (indicated by a cross). Further details in Figure S4 supplemental information).

The feasibility of time-gated Raman spectral multiplexing to measure 3-D Raman images of turbid samples was then investigated using a new sample consisting of two polystyrene blocks placed on Teflon layers of $0.6 \mathrm{~mm}$ and $1.5 \mathrm{~mm}$ thickness (see schematic in Figure 3a).

Previous works on depth profiling using time-gated Raman reported a $x-y$ spatial resolution of $\sim 1 \mathrm{~mm}$ for Teflon surface layers of $1 \mathrm{~mm}$ using defocused $400 \mathrm{~nm}$ excitation, ${ }^{6}$ suggesting an adequate level of spatial resolution was possible. Here, raster scanning in the $x-y$ plane using $250 \mu \mathrm{m}$ steps size ( $40 \times 40$ pixels) and two data sets were acquired for each pixel by alternatively switching the DMD mirrors to select the spectral bands of the two materials, Teflon and PS. The integration times for each material were 1s for Teflon and $15 \mathrm{~s}$ for PS respectively. Time-gated Raman maps of both the polystyrene (PS) and the Teflon are presented in Figure $3 \mathrm{~b}$ for a selection of gate times selected to highlight the time evolution of the signal (data arranged into 80_ps bin widths). The results show that the Raman signal from the Teflon surface layer peaks around $140 \mathrm{ps}$. At this time point, the signal from polystyrene is detectable in the region corresponding to the thinner Teflon layer. The signal from the 
polystyrene on this region peaks at $\sim 200 \mathrm{ps}$ and finally, the signal from the polystyrene through the thicker layer peaks at $\sim 250 \mathrm{ps}$. Applying the calibration formula determined above, the value of the Teflon thickness is estimated to depths of $511 \pm 270 \mu \mathrm{m}$ and $938 \pm 360 \mu \mathrm{m}$ for the two layers of Teflon (actual measured depths were $600 \mu \mathrm{m}$ and $1500 \mu \mathrm{m}$ ). Using the photon time-of-flight at each pixel, we proceed in reconstructing a 3D map of the sample. For each pixel, the time at which the maximum intensity of both the polystyrene and Teflon signals $I(t)$ occurs is determined and maps produced based upon the mean photon time of arrival (Figure $3 \mathrm{c}$ for polystyrene map). The Teflon map determines the zero point (surface) and the two data sets are combined to create a 3D map of the binary sample (Figure 3d).

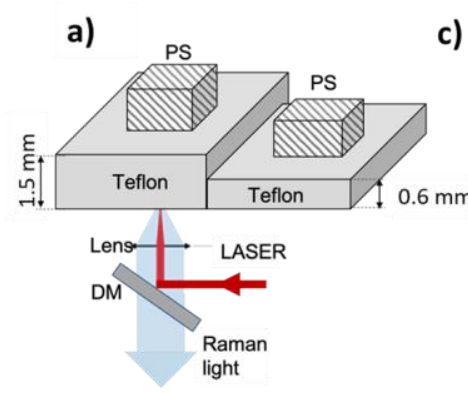

c)

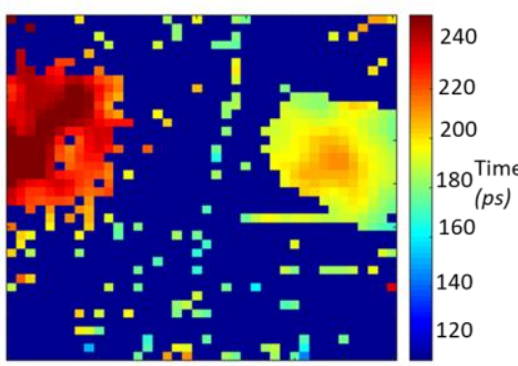

b)
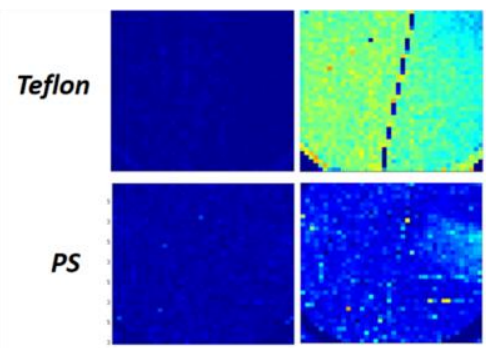

$60 p s$
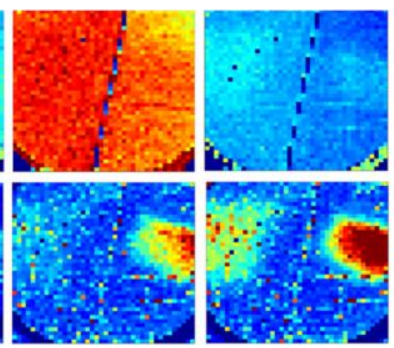

$\begin{array}{ll}140 p s & 200 p s \\ \text { (Peak of surface layer) } & \text { (Peak of signal }\end{array}$

through thin layer)

\section{Time}
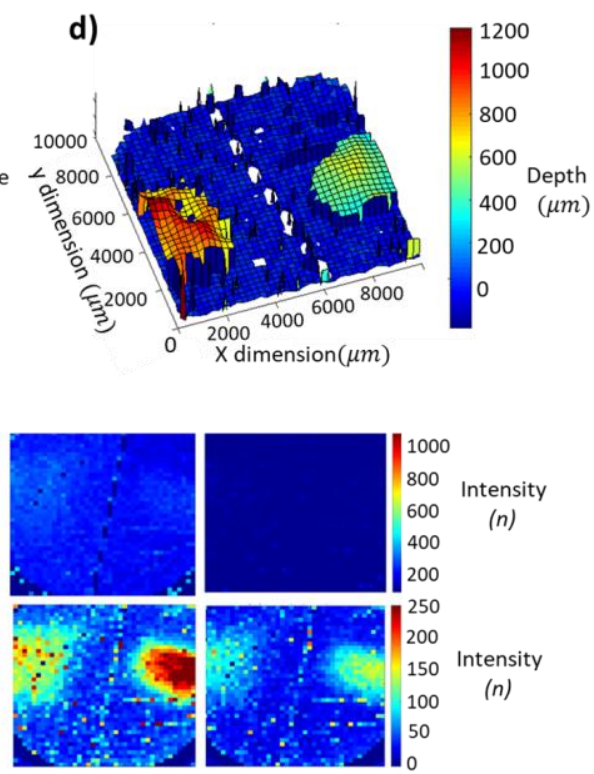

250ps

(Peak of signal

through thick layer)

500ps

Figure 3. a) Schematic description of the Teflon/Polystyrene sample. b) 2D time gated Raman maps of both Teflon and polystyrene (PS) generated by multiplexing the bands shown in Figure $2 \mathrm{a}$ (red for Teflon, blue for PS). Gate times are selected to show the time evolution of the signal. c) 2D map showing the diffusion time for the PS signal. For each pixel, the time of maximum Raman intensity of polystyrene was used to create a Raman photon time of flight map. d) Depth surface plots of both Teflon and polystyrene generated by calculating the depth from the mean time of flight at each pixel. The data is overlaid to represent the spatial positions of the sample with the Teflon surface shown at zero depth (blue).

\section{Time-gated Raman for fluorescence suppression of pigmented samples relevant to cultural heritage.}

Here we investigated the feasibility of combining time-gated Raman spectral multiplexing with micro-SORS to obtain sub-surface molecular analysis from samples eliciting strong fluorescence backgrounds. 
As the focus here has been on samples relevant to Cultural Heritage applications, we first investigated the ability of the time-gated Raman instrument to measure Raman spectra of several common red pigments. Red lake, Carmine Naccarat, Quinacridone Red Magenta and fluorescein were selected as they are particularly difficult to analyze using CW Raman techniques. For each sample, the DMD was programmed to select the full spectral range (750 $-1650 \mathrm{~cm}^{-1}$ ), the time profile $I(t)$ was then measured for all samples to determine the lifetimes of the fluorescence emission. Although the excitation wavelength was longer than the peaks of the absorption bands, strong fluorescence emission was observed for all these pigment molecules. Therefore, the excitation mechanism is likely to be two-photon excitation. Normalized $I(t)$ for all pigments are shown in Figure S2 Supplemental material. All pigments had fluorescence lifetimes in the range 100-180 ps with the exception of fluorescein, for which the lifetime was calculated as 520 ps.

Time-gated Raman spectra using the DMD in scanning mode were recorded from all pigment samples to establish their main Raman bands and to estimate the level of fluorescence suppression that could be achieved with the current instrument. The data in Figure 4 is presented as progressively increasing gate widths to allow a comparison between time gating and a steady state CCD based system. Figure 4a presents the time-gated Raman spectra of Red lake showing that for long gate times (>550 ps, equivalent to continuous wave Raman spectroscopy) a Raman band at $1460 \mathrm{~cm}^{-1}$ is weakly observed through the intense fluorescence background. For short gate times (e.g. 80-150 ps), Raman bands at $1000 \mathrm{~cm}^{-1}, 1100 \mathrm{~cm}^{-1}, 1460$ $\mathrm{cm}^{-1}$ and $1230 \mathrm{~cm}^{-1}$ can be observed, in agreement with Raman spectra reported previously, recorded with a FT Raman spectrometer based on 1064 nm laser excitation. ${ }^{37}$

The time-gated Raman spectra of Carmine Naccarat presented in Figure 4b. For continuous wave (long time gates of $550 \mathrm{ps}$ ) Raman bands at $1314 \mathrm{~cm}^{-1}$ and $1255 \mathrm{~cm}^{-1}$ are weakly observed. For short gate times (e.g. 80 ps), the reduced fluorescence background enhances the relative intensities of these features, allowing also detection of weaker Raman bands at 1110 $\mathrm{cm}^{-1}$ and $1430 \mathrm{~cm}^{-1}$. These features are in agreement with FT Raman by Burgio and Clark ${ }^{42}$. Figure 4c presents the Raman spectra of quinacridone Red Magenta. Figure 4c (550 ps) shows that for long gate times (CW), the Raman band at $1316 \mathrm{~cm}^{-1}$ is the only spectral feature observable. For short gate times (Figure $4 \mathrm{c} 80 \mathrm{ps}$ ), the $1316 \mathrm{~cm}^{-1}$ band is greatly enhanced and spectral features at $1508 \mathrm{~cm}^{-1}, 1570 \mathrm{~cm}^{-1}, 1592 \mathrm{~cm}^{-1}$ and $1645 \mathrm{~cm}^{-1}$ are observed, in agreement with FT Raman spectra recorded by Burgio and Clark ${ }^{42}$.

Time-gated Raman spectra of fluorescein are presented in Figure 4d. With a fluorescence lifetime of $520 \mathrm{ps}$, the time-gated detection provides the most efficient fluorescence rejection among all pigment samples. For long gate times (Figure $4 \mathrm{~d}(550 \mathrm{ps})$ ), the Raman bands at $1180 \mathrm{~cm}^{-1}$ and $1316 \mathrm{~cm}^{-1}$ are just observable though the intense fluorescent background. At shorter gate times (Figure 4d, $80 \mathrm{ps)} \mathrm{the} \mathrm{level} \mathrm{of} \mathrm{fluorescence} \mathrm{suppression} \mathrm{is} \mathrm{very} \mathrm{efficient} \mathrm{and}$ enables detection of Raman bands at $1170 \mathrm{~cm}^{-1}, 1318 \mathrm{~cm}^{-1}, 1396 \mathrm{~cm}^{-1}, 1484 \mathrm{~cm}^{-1}$ and 1571 $\mathrm{cm}^{-1}$ which are in good agreement with previous works by Wang et al. ${ }^{43}$ 

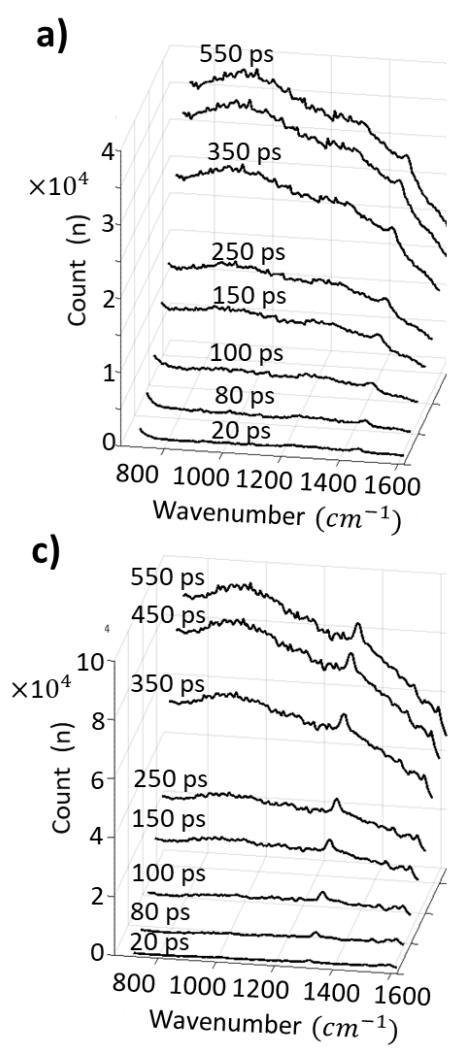
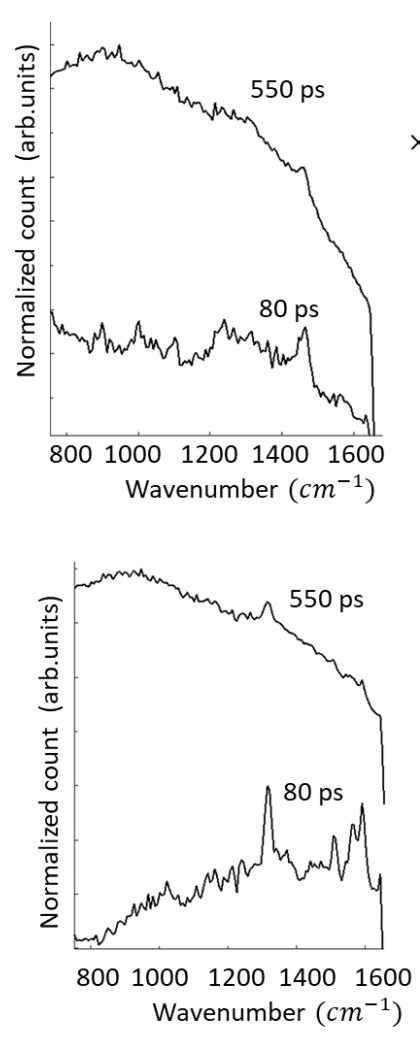

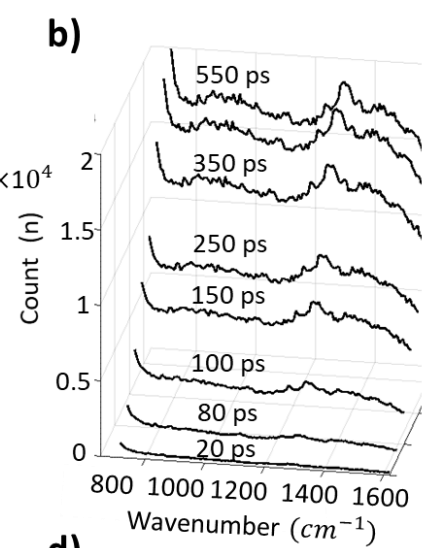

d)

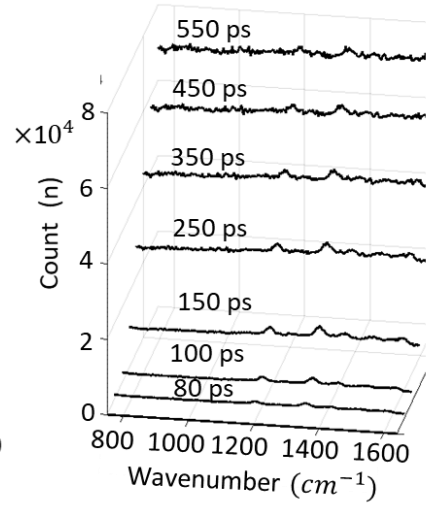

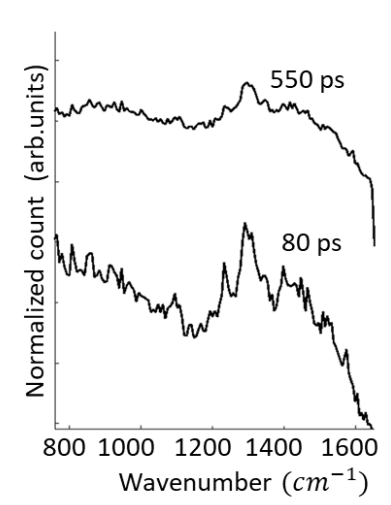

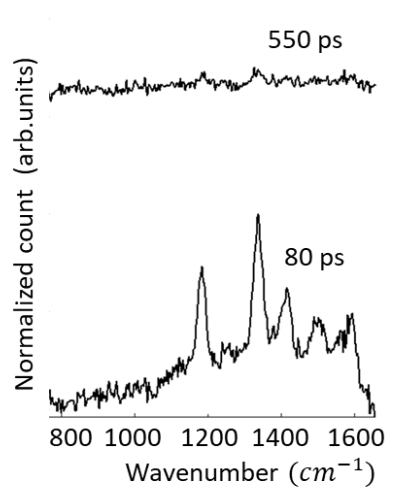

Figure 4. Time-gated Raman spectra of red pigments: a) Red lake pigment. b) Carmine Naccarat. c) Quinacridone Red Magenta. d) fluorescein. Times indicate width of gate window for comparison between steady state CCD based systems ( 0 ps corresponds to time the first Raman photons are detected).

To estimate the performance of fluorescence suppression, a simple single exponential model proposed by Everall et al ${ }^{44}$ was used based on the ratios of the fluorophores lifetime and the width of the time gate (equation 1). This simple model has been shown to provide results consistent with experiment for Kerr gated and iCCD based time gated Raman systems. ${ }^{8,44}$

$f l_{b g} /_{\text {Raman }}=\frac{\int_{0}^{\infty} e^{-t / t_{f}} d t}{\int_{0}^{t_{g} e^{-t / t_{f}} d t}}=\frac{1}{1-e^{-t_{g} / t_{f}}} \approx \frac{t_{f}}{t_{g}} \quad$ [Eq1],

where $t=$ time, $t_{f}=$ fluorescence lifetime and $t_{g}$ is gate width.

If we assume our minimum gate width is equal to the FWHM of the instrument response function, $60 \mathrm{ps}$, and take the lifetimes as measured from the emission curves shown in Figure S2, we can calculate the expected amount of fluorescence suppression using Equation 1.

We then evaluate the performance of the time gated Raman instrument by measuring the improvement factor in the Raman/fluorescence background by comparing the intensity ratios between long $(\mathrm{CW})$ and optimized time gates. The measured Raman/fluorescence background ratio is calculated by integrating the Raman signal above and below the background level of the most prominent Raman peak. The measured Raman/fluorescence background ratio vs time gate data is shown in Figure S3 in the Supplemental material. The expected and measured improvements along with the lifetimes are shown below in Table 1. 
Table 1. Lifetimes predicted and measured improvement of FL/Raman.

\begin{tabular}{|l|l|l|l|}
\hline Sample & $\begin{array}{l}\text { Lifetime } \\
(\mathbf{p s})\end{array}$ & $\begin{array}{l}\text { Calculated } \\
\text { Improvement } \\
\text { Raman/fl background }\end{array}$ & $\begin{array}{l}\text { Measured } \\
\text { improvement } \\
\text { Raman/fl background }\end{array}$ \\
\hline Red lake & 106 & 2.3 & 2.8 \\
\hline Carmine Naccarat & 137 & 2.8 & 2.6 \\
\hline Quinacridone Red Magenta & 183 & 3.6 & 3.6 \\
\hline Fluorescein & 520 & 9.1 & 11.7 \\
\hline
\end{tabular}

\section{Time-gated micro-SORS for sub-surface analysis of materials with strong fluorescence backgrounds with a non-fluorescing surface layer}

After establishing the main bands in the Raman spectra of the four red pigments, layered samples using fluorescein as a sub-surface layer were prepared to investigate the feasibility of using micro-SORS combined with time-gating to recover the Raman signal from fluorescing sublayer. Although micro-SORS (as well as SORS) can be used to suppress fluorescence from overlayers it cannot deal with fluorescence originating from the target sublayer. ${ }^{30}$ As such, being able to address this analytical situation in cultural heritage is of high importance. Without loss of generality, fluorescein was selected here for demonstration of proof-ofprinciple, as it has the longest fluorescence lifetime out of all pigments, thus enabling the most efficient fluorescence suppression by time-gated detection.

First, a test sample was prepared with approximately $1 \mathrm{~mm}^{3}$ of fluorescein powder, layered upon a $600 \mu \mathrm{m}$ thick layer of Teflon (eight layers of PTFE tape). The intensity as a function of time $I(t)$ was measured separately for the Teflon, fluorescein alone and fluorescein through $600 \mu \mathrm{m}$ of Teflon, where the measured signal consists of all excited photons, both Raman and fluorescence by multiplexing the Raman bands specific to each material. The data is combined and presented in Figure 5a. By comparing the data in Figure 5a it is observed although the fluorescein signal is delayed due to photon diffusion the first $100 \mathrm{ps}$ of fluorescein signal overlaps temporally with the Teflon signal from the surface layer; consequently, time-gating alone cannot separate the sublayer Raman signal from the signal generated at the surface. Using a Nikon 20x 0.75 NA microscope objective, Raman spectra were acquired with the sample in focus and defocused by $-200 \mu \mathrm{m}$ and $-400 \mu \mathrm{m}$ (Figures $5 \mathrm{~b}, \mathrm{c} \& \mathrm{~d}$ ).

Figure $5 \mathrm{~b}$ shows that when the sample is in focus, the Raman signal from the Teflon surface layer dominates the Raman signals at short gate times (Figure $5 \mathrm{~b} \mathrm{e.g.} 80 \mathrm{ps}$ ). With the gate time optimized for maximum signal from fluorescein (140 ps), the Teflon signal is still observed and the Raman band of fluorescein at $1170 \mathrm{~cm}^{-1}$ is just visible. For long gate widths (>500 ps) the intense fluorescence background dominates and detection of any Raman signal of fluorescein is not possible. Figure 5c shows that defocusing the objective by $-200 \mu \mathrm{m}$ leads to an attenuation of the signal from the Teflon surface layer. The highest intensity of fluorescein signal was observed at $140 \mathrm{ps}$ (Figure 5c), but still the $1318 \mathrm{~cm}^{-1}$ peak is barely detectable. No Raman bands are observed for long time-gates (> 500ps). When the sample was defocused further to $-400 \mu \mathrm{m}$, the signal of Teflon surface was again attenuated at short gate times (Figure 5d, e.g. $80 \mathrm{ps}$ ), but provided the best detection of Raman bands of fluorescein at $1180 \mathrm{~cm}^{-1}$ and $1318 \mathrm{~cm}^{-1}$ at time-gates of $140 \mathrm{ps}$ (Figure 5d). Once again, for long gate widths, the intense fluorescence background dominates, and no Raman can be retrieved.

These results demonstrate that time-gated micro-SORS provides the ability to attenuate the signal from the surface layer while allowing discrimination between Raman and fluorescence photons from the sublayer. 

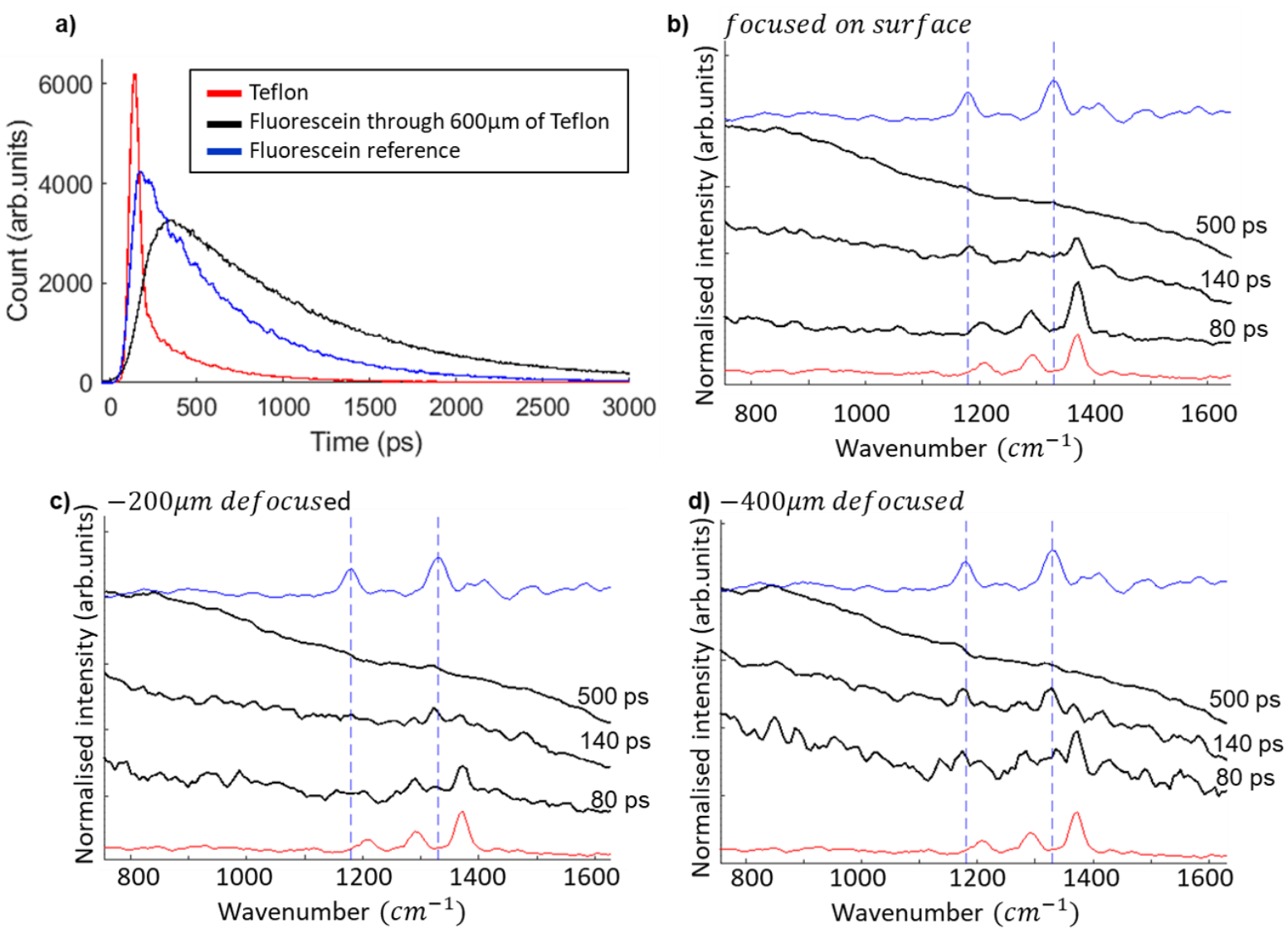

Figure 5. a) Composite plot combining the emission intensity as a function of time for Teflon and fluorescein reference samples and a fluorescein through $600 \mu \mathrm{m}$ of Teflon. Data acquired by multiplexing the $1216 \mathrm{~cm}^{-1}, 1299 \mathrm{~cm}^{-1}$ and $1379 \mathrm{~cm}^{-1}$ bands for Teflon, and the $1180 \mathrm{~cm}^{-1}$ and $1318 \mathrm{~cm}^{-1}$ bands for fluorescein. By comparison of the blue and black curves it is observed that although the fluorescein signal is delayed due to the photon diffusion process there is still overlap between the signal generated at the surface and the leading edge of the fluorescein signal. b) In focus time-gated Raman spectra. c). micro-SORS time-gated Raman spectra with $-200 \mu \mathrm{m}$ defocus. d) micro-SORS time-gated Raman spectra with $-400 \mu \mathrm{m}$ defocus. Blue dashed vertical lines at $1180 \mathrm{~cm}^{-1}$ and $1318 \mathrm{~cm}^{-1}$ are guiding lines for the reference fluorescein bands.

\section{Time-gated micro-SORS for sub-surface analysis of materials with strong fluorescence backgrounds with a highly fluorescing surface layer}

The temporal overlap between the surface and the sublayer signals can be even more accentuated when both surface layer and sub-surface elicit strong fluorescence background. This most difficult case was investigated here by preparing a sample of $1 \mathrm{~mm}^{3}$ of fluorescein powder layered behind top of a $100 \mu \mathrm{m}$ thick layer of highly fluorescent brown paper. The time-gated gated Raman spectra were acquired with the sample in focus and then $400 \mu \mathrm{m}$ defocused in order to take advantage of the micro-SORS effect. With the microscope focused on the surface of the sample, for short gate times (Figure 6a, e.g. 130 ps), the fluorescence background from the brown paper surface layer dominates the signal and the fluorescein 
spectrum is barely observed. At longer gate times (Figure 6a, e.g. 500 ps) the intense fluorescence background, from both the surface and sublayers, dominates and no Raman spectral bands were observed.

However, a significant enhancement in the detection of Raman bands of fluorescein was observed when the microscope was defocused by $400 \mu \mathrm{m}$ and short gate times were selected for detection (Figure 6b, e.g. 130 ps). In this case, the signal from the surface layer of brown paper was sufficiently suppressed coupled with an enhancement of the sublayer to allow successful recovery of the fluorescein Raman signal. For longer gate widths (> $500 \mathrm{ps})$, the intense fluorescence background dominated, and again, no Raman spectral features were observed.
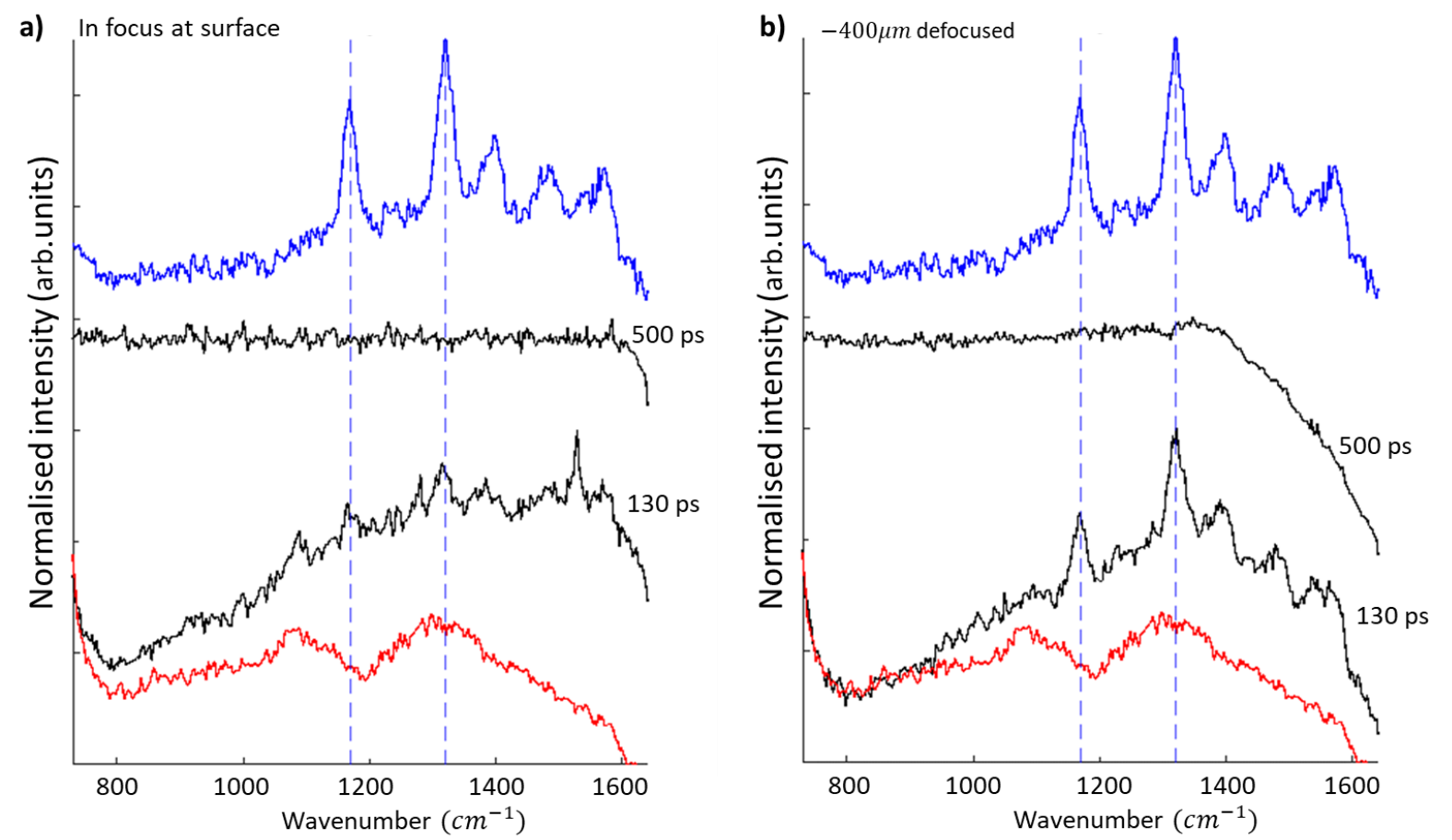

Figure 6. Micro-SORS time-gated Raman spectra of a layered sample consisting of $100 \mu \mathrm{m}$ thick brown paper surface layer and fluorescein sublayer. a) Laser focused on the paper surface. b) $-400 \mu \mathrm{m}$ defocused. Time gated reference spectra of fluorescein and brown paper are shown in blue and red. Raman spectra at 130 ps time-gate (highest intensity of fluorescein Raman signals) are compared to Raman spectra at 500 ps time-gate.

These results demonstrate that the combination of time-gated and micro-SORS is a powerful approach for Raman analysis of subsurface fluorescing materials. Although the defocusing micro-SORS used here led to a decrease in the signal to noise ratio, it is possible to employ more signal-efficient micro-SORS techniques, such as DMD-based micro-SORS to generate ring collection geometries. These techniques could significantly increase the optical throughput and decrease the acquisition times, as required for real-world applications.

\section{Conclusion}

By considering scattered photons through diffusive media we have applied time-gated and spatially-offset Raman spectroscopy to obtain sub surface molecular analysis and imaging for both fluorescing and non-fluorescing samples. The multiplexed spectral detection enabled by 
a digital micromirror device (DMD) allowed fast acquisition of the time-gated signals to enable 3D Raman maps (raster scanning in the lateral $x$ - $y$ plane and use time-of-flight calibration for the axial $z$-direction). Utilizing the signal-to-noise advantage obtained by spectral multiplexing and operating in a time correlated single photon counting (TCSPC) mode, sub millimetre resolution molecular depth mapping can be achieved with dwell times on the order of seconds per pixel. Further improvements in the depth accuracy could be made by improving the calibration (machining more precise uniform calibration blocks) and the data analysis methods (fitting a more complex model to the data to account for scattering attenuation).

Defocusing and scattering of the excitation beam and Raman scattered light limits the lateral resolution for depth measurements. However, because of this, further speed improvements could be made by using a higher laser power without risk of laser damage to the sample (50 $\mathrm{mW}$ laser power was used for the depth measurements).

Time-gated Raman spectroscopy was successfully applied to record Raman spectra common red pigments encountered in cultural heritage. In spite of the very short fluorescence lifetimes in the 100-550 ps range, the instrument provided efficient rejection of the fluorescence background enabling detection of the main Raman bands.

Finally, we combined time-gating and micro-SORS to recover the Raman signals of red pigments placed behind layers of optically turbid materials. Using defocusing micro-SORS, both fluorescence and Raman signals from the surface layers were further suppressed to enable enhanced detection of the Raman signals from the deeper sublayer. Although the spectral multiplexing enabled by the DMD allowed sufficient detection of the Raman signals, further improvements in the data acquisition speed could be made using DMD-based micro-SORS techniques that have typically higher optical throughput. ${ }^{35}$ Such techniques can reduce the acquisition times to levels required for real-life applications.

The combination of a single pixel, cooled detector operating in TCSPC mode with a DMD as the wavelength selective component provides a solution to many of the technical limitations current time gating techniques face. The DMD has a high fill factor of $92 \%$ and multiplexing provides a significant signal to noise advantage. The gated dark count rate of the detector is significantly less than the continuous dark count $(<0.5 \mathrm{cps}$ gated). The single pixel SPAD coupled with optimized optics and 30 ps laser pulse results in an IRF FWHM of 60 ps for the whole instrument - essential for effective suppression of short-lived fluorophores and highresolution depth measurements. In conclusion, we showed that time-gated Raman spectroscopy based on multiplexed detection, and combination with micro-SORS, is a powerful technique for subsurface molecular analysis and imaging, which can find practical applications in medical imaging, art and cultural heritage, forensics, and industry.

\section{Disclaimer}

The authors declare no conflicts of interest.

\section{Acknowledgements}

This work was supported by the Engineering and Physical Sciences Research Council [grant number EP/L025620/1].

\section{References}

1. $\quad$ D.W.S. Shipp, F.S. Sinjab, I. Notingher. "Raman spectroscopy : Techniques and applications in the life sciences". 2017. Adv. Opt. Photon. 9, 315-428 (2017).

2. N.J. Everall. "Confocal Raman microscopy: Common errors and artefacts". Analyst. 
2010. 135(10): 2512-2522. 10.1039/c0an00371a.

3. Z. Liao, F. Sinjab, H.M. Elsheikha, I. Notingher. "Optical sectioning in multifoci Raman hyperspectral imaging”. J. Raman Spectrosc. 2018. 49(10): 1660-1667. 10.1002/jrs.5450.

4. P.D.A. Pudney, T.M. Hancewicz, D.G. Cunningham, M.C. Brown. "Quantifying the microstructures of soft solid materials by confocal Raman spectroscopy". Vib. Spectrosc. 2004. 34(1): 123-135. 10.1016/j.vibspec.2003.08.006.

5. J. Kekkonen, J. Nissinen, I. Nissinen. "Depth Analysis of Semi-Transparent Media by a Time-Correlated CMOS SPAD Line Sensor-Based Depth-Resolving Raman Spectrometer". IEEE Sens. J. IEEE, 2019. 19(16): 6711-6720.

10.1109/JSEN.2019.2913222.

6. F. Ariese, H. Meuzelaar, M.M. Kerssens, J.B. Buijs, C. Gooijer. "Picosecond Raman spectroscopy with a fast intensified CCD camera for depth analysis of diffusely scattering media". 2009. Analyst. 134(6): 1192-1197. 10.1039/b821437a.

7. S.Konugolu.Venkata. Sekar, S. Mosca, S. Tannert, G. Valentini, F. Martelli, Tiziano Binzoni, Y. Prokazov et al. "Time domain diffuse Raman spectrometer based on a TCSPC camera for the depth analysis of diffusive media." Optics letters. (2018) 43(9): 2134-2137.10.1364/OL.43.002134.

8. E. V Efremov, J.B. Buijs, C. Gooijer, F. Ariese. "Fluorescence Rejection in Resonance Raman Spectroscopy Using a Picosecond-Gated Intensified Charge-Coupled Device Camera". 2007. Applied spectroscopy. 61(6): 571-578. 10.1366/000370207781269873.

9. P. Matousek, M. Towrie, A.W. Parker, C.L. Facility, C. Rutherford, C. Ma, et al. "Fluorescence Suppression in Raman Spectroscopy using a High Performance Picosecond Kerr Gate". 2001. Journal of Raman Spectroscopy. 32(12): 983-988. 10.1002/jrs.784.

10. T. Rojalin, L. Kurki, T. Laaksonen, T. Viitala, J. Kostamovaara, K.C. Gordon, et al. "Fluorescence-suppressed time-resolved Raman spectroscopy of pharmaceuticals using complementary metal-oxide semiconductor (CMOS) single-photon avalanche diode (SPAD) detector". Anal. Bioanal. Chem. 2016. 408(3): 761-774. 10.1007/s00216-015-9156-6.]

11. A. Usai, N. Finlayson, C.D. Gregory, C. Campbell, R.K. Henderson. "Separating fluorescence from Raman spectra using a CMOS SPAD TCSPC line sensor for biomedical applications". In Optical Biopsy XVII: Toward Real-Time Spectroscopic Imaging and Diagnosis, vol. 10873, p. 108730R. International Society for Optics and Photonics, 2019. 26. 10.1117/12.2508459.

12. J. Kostamovaara, J. Tenhunen, M. Kögler, I. Nissinen, J. Nissinen, P. Keränen. "Fluorescence suppression in Raman spectroscopy using a time-gated CMOS SPAD." Opt. Express. 2013. 21(25): 31632-31645. 10.1364/OE.21.031632.

13. P. Matousek, N. Everall, M. Towrie, A.W. Parker. "Depth profiling in diffusely scattering media using Raman spectroscopy and picosecond Kerr gating". Appl. Spectrosc. 2005. 59(2): 200-205. 10.1366/0003702053085115.

14. F. Sinjab, Z. Liao, I. Notingher. "Applications of Spatial Light Modulators in Raman Spectroscopy". Applied spectroscopy 2019. 73(7): 727-746. 10.1177/0003702819834575.

15. D. Cebeci, B. Mankani, D. Ben-Amotz. "Recent Trends in Compressive Raman Spectroscopy Using DMD-Based Binary Detection”. J. Imaging. 2018. 5(1): 1. 10.3390/jimaging5010001.

16. C. Scotté, H.B. De Aguiar, D. Marguet, E.M. Green, P. Bouzy, S. Vergnole, et al. "Assessment of Compressive Raman versus Hyperspectral Raman for 
Microcalcification Chemical Imaging”. Anal. Chem. 2018. 90(12): 7197-7203. 10.1021/acs.analchem.7b05303.

17. D.S. Wilcox, G.T. Buzzard, B.J. Lucier, O.G. Rehrauer, P. Wang, D. Ben-Amotz. "Digital compressive chemical quantitation and hyperspectral imaging". Analyst. 2013. 138(17): 4982. 10.1039/c3an00309d.

18. O.G. Rehrauer, S. Claridge. "Multivariate Statistical Methods that Enable Fast Raman Spectroscopy". 2016. PhD thesis Purdue University.

19. F. Sinjab, K. Kong, G. Gibson, S. Varma, H. Williams, I. Notingher. "Tissue diagnosis using power-sharing multifocal Raman micro-spectroscopy and auto- fluorescence imaging”. 2016. Biomedical optics express, 7(8): 2993-3006. 10.1364/BOE.7.002993

20. F. Sinjab, D. Awuah, G. Gibson, M. Padgett, A.M. Ghaemmaghami, I. Notingher. "Holographic optical trapping Raman micro-spectroscopy for non-invasive measurement and manipulation of live cells". Opt. Express. 2018. 26(19): 25211. 10.1364/oe.26.025211.

21. L. Collard, F. Sinjab, I. Notingher. "Raman Spectroscopy Study of CurvatureMediated Lipid Packing and Sorting in Single Lipid Vesicles". Biophys journal 2019. 117(9): 1589-1598. 10.1016/j.bpj.2019.09.020.

22. F. Sinjab, H.M. Elsheikha, M. Dooley, I. Notingher. "Induction and measurement of the early stage of a host-parasite interaction using a combined optical trapping and Raman microspectroscopy system". J. Biophotonics. 2020. 13(2): 1-8. 10.1002/jbio.201960065.

23. Z. Liao, F. Sinjab, A. Nommeots-Nomm, J. Jones, L. Ruiz-Cantu, J. Yang, et al. "Feasibility of Spatially Offset Raman Spectroscopy for in Vitro and in Vivo Monitoring Mineralization of Bone Tissue Engineering Scaffolds". Anal. Chem. 2017. 89(1): 847-853. 10.1021/acs.analchem.6b03785.

24. Z. Liao, F. Sinjab, G. Gibson, M. Padgett, I. Notingher. "DMD-based softwareconfigurable spatially-offset Raman spectroscopy for spectral depth-profiling of optically turbid samples”. Opt. Express. 2016. 24(12): 12701. 10.1364/OE.24.012701.

25. M. Dooley, A. Prasopthum, Z. Liao, F. Sinjab, J. McLaren, F.R.A.J. Rose, et al. "Spatially-offset Raman spectroscopy for monitoring mineralization of bone tissue engineering scaffolds: feasibility study based on phantom samples". Biomed. Opt. Express. 2019. 10(4): 1678. 10.1364/boe.10.001678.

26. E. Draper, M. Towrie, M.D. Morris, A.W. Parker, P. Matousek, I.P. Clark, et al. "Numerical Simulations of Subsurface Probing in Diffusely Scattering Media Using Spatially Offset Raman Spectroscopy”. Appl. Spectrosc. 2005. 59(12): 1485-1492. 10.1366/000370205775142548.

27. Z. Wang, H. Ding, G. Lu, X. Bi. "Use of a mechanical iris-based fiber optic probe for spatially offset Raman spectroscopy”. Opt. Lett. 2014. 39(13): 3790. 10.1364/OL.39.003790.

28. J. Qin, M.S. Kim, W.F. Schmidt, B.K. Cho, Y. Peng, K. Chao. “A line-scan hyperspectral Raman system for spatially offset Raman spectroscopy". J. Raman Spectrosc. 2016. 47(4): 437-443. 10.1002/jrs.4825.

29. M. V. Schulmerich, J.H. Cole, K.A. Dooley, M.D. Morris, J.M. Kreider, S.A. Goldstein, et al. "Noninvasive Raman tomographic imaging of canine bone tissue". J. Biomed. Opt. 2008. 13(2): 020506. 10.1117/1.2904940.

30. C. Conti, A. Botteon, C. Colombo, M. Realini, P. Matousek. "Fluorescence suppression using micro-scale spatially offset Raman spectroscopy". Analyst. 2016. 141(18): 5374-5381. 10.1039/c6an00852f.

31. O.G. Rehrauer, B.R. Mankani, G.T. Buzzard, B.J. Lucier, D. Ben-Amotz. "Fluorescence modeling for optimized-binary compressive detection Raman 
spectroscopy". Opt. Express. 2015. 23(18): 23935. 10.1364/OE.23.023935.

32. B.M. Davis, A.J. Hemphill, D. Cebeci Maltaş, M.A. Zipper, P. Wang, D. Ben-Amotz. "Multivariate hyperspectral raman imaging using compressive detection". Anal. Chem. 2011. 83(13): 5086-5092. 10.1021/ac103259v.

33. O.G. Rehrauer, V.C. Dinh, B.R. Mankani, G.T. Buzzard, B.J. Lucier, D. Ben-Amotz. "Binary Complementary Filters for Compressive Raman Spectroscopy". Appl. Spectrosc. 2018. 72(1): 69-78. 10.1177/0003702817732324.

34. C.J. Corden, D.W. Shipp, P. Matousek, I. Notingher. "Fast Raman spectral mapping of highly fluorescing samples by time-gated spectral multiplexed detection". Opt. Lett. 2018. 43(23): 5733. 10.1364/OL.43.005733.

35. C. Conti, A. Botteon, C. Colombo, M. Realini, P. Matousek. "Fluorescence suppression using micro-scale spatially offset Raman spectroscopy". Analyst. 2016. 141(18): 5374-5381. 10.1039/c6an00852f.

36. R.D. Harley. Artists' pigments c.1600-1835: a study in English documentary sources. Archetype Publications, London, 2001. viii.

37. C.M. Schmidt, K.A. Trentelman. "1064 nm DISPERSIVE RAMAN MICROSPECTROSCOPY FOR THE IN-SITU IDENTIFICATION OF ORGANIC RED COLORANTS”. e-Preservation Sci. 2009. 6: 10-21.

38. Carle, Reinhold, and Ralf Schweiggert, eds. "Handbook on natural pigments in food and beverages: Industrial applications for improving food color. Woodhead Publishing, 2016.

39. S.Q. Lomax. "Phthalocyanine and quinacridone pigments: their history, properties and use". Stud. Conserv. 2005. 50(sup1): 19-29. 10.1179/sic.2005.50.Supplement-1.19.

40. I.E. Iping Petterson, P. Dvoák, J.B. Buijs, C. Gooijer, F. Ariese. "Time-resolved spatially offset Raman spectroscopy for depth analysis of diffusely scattering layers". Analyst. 2010. 135(12): 3255-3259. 10.1039/c0an00611d.

41. N. Everall, T. Hahn, P. Matousek, A.W. Parker, M. Towrie. "Photon migration in Raman spectroscopy”. Appl. Spectrosc. 2004. 58(5): 591-597. 10.1366/000370204774103426.

42. L. Burgio, R.J.H. Clark. Library of FT-Raman spectra of pigments, minerals, pigment media and varnishes, and supplement to existing library of Raman spectra of pigments with visible excitation. Mol. Biomol. Spectrosc. 2001. 57(7): 1491-1521. 10.1016/S1386-1425(00)00495-9.

43. L. Wang, A. Roitberg, C. Meuse, A.K. Gaigalas. "Raman and FTIR spectroscopies of fluorescein in solutions". Spectrochim. Acta - Part A Mol. Biomol. Spectrosc. 2001. 57(9): 1781-1791. 10.1016/S1386-1425(01)00408-5.

44. N. Everall, T. Hahn, P. Matousek, A.W. Parker, M. Towrie. "Picosecond timeresolved Raman spectroscopy of solids: Capabilities and limitations for fluorescence rejection and the influence of diffuse reflectance". Appl. Spectrosc. 2001. 55(12): 1701-1708. 10.1366/0003702011954053.

45. M. V. Schulmerich, W.F. Finney, R.A. Fredricks, M.D. Morris. "Subsurface Raman spectroscopy and mapping using a globally illuminated non-confocal fiber-optic array probe in the presence of Raman photon migration". Appl. Spectrosc. 2006. 60(2): 109114. 10.1366/000370206776023340.

46. Z. Liao, F. Sinjab, G. Gibson, M. Padgett, I. Notingher. "DMD-based softwareconfigurable spatially-offset Raman spectroscopy for spectral depth-profiling of optically turbid samples”. Opt. Express. 2016. 24(12): 12701. 10.1364/OE.24.012701.

47. P. Matousek, C. Conti, C. Colombo, M. Realini. "Monte Carlo simulations of subsurface analysis of painted layers in micro-scale spatially offset Raman spectroscopy”. Appl. Spectrosc. 2015. 69(9): 1091-1095. 10.1366/15-07894. 
48. C. Conti, M. Realini, C. Colombo, K. Sowoidnich, N.K. Afseth, M. Bertasa, et al. "Noninvasive analysis of thin turbid layers using microscale spatially offset Raman spectroscopy". Anal. Chem. 2015. 87(11): 5810-5815.

10.1021/acs.analchem.5b01080. 